\title{
Relation of parallel resistance to the passive double SAW resonator
}

\begin{abstract}
This paper presents the relationship of parallel resistor to the frequency response of the passive remote acoustic wave resonators (SAWRs) sensor system in $433.42 \mathrm{MHz}$ and 433.92MHz. Impedance matching is achieved with the connection of L-network to the parallel SAW resonator. The main objective of this finding is to improve the sensor of narrow bandwidth application. Circuit with high quality factor ( $\mathrm{Q}$ factor) has better suppression for narrow band application. Parallel resistor improves the system by increasing the $\mathrm{Q}$ factor. Increasing the parallel resistance will decreased the bandwidth of the resonant frequency. Simulation results of the system are presented and discussed.
\end{abstract}

Keyword: L-network; Quality factor; SAWR sensor system 